\title{
Coherent control of excited state populations in rubidium using Rabi oscillations.
}

\author{
D A Malik, A V Kimel and W J van der Zande. \\ Radboud University Nijmegen, Institute for Molecules and Materials, P.O. Box 9010, NL-6500 GL \\ Nijmegen, The Netherlands \\ E-mail:w.vanderzande@science.ru.nl
}

\begin{abstract}
Complete population inversion is demonstrated analytically in the strong field limit in a three level system using the rubidium 5s-5p-5d transition. We exploit the pre-transients of an amplitude shaped laser pulse to direct the dynamics of the Rabi oscillations such that the population transfer to the excited state can be controlled in a wide range from a strongly enhanced to vanishing one. The excitation is performed under off-resonant conditions. During the intense ultrafast pulse levels shift to such an extent that two-photon resonant excitation becomes possible, even though a frequency spectrum does not contain photons with energies necessary for excitation of an unperturbed system. Within the reach of experimental parameters we demonstrate $70 \%$ population transfer.
\end{abstract}

PACS Codes: 32.80.Qk, 32.80.Wr

\section{Introduction}

The recent developments of ultrafast lasers and modern pulse shapers, which can change the amplitude, phase and polarization of all individual frequency components of a fast pulse, has made coherent control a rapidly growing field in contemporary science. Many demonstrations of coherent control take place under conditions of multi-photon excitation with little population 
transfer. Under these conditions, perturbation theory applies and the interaction can be described in the frequency domain. As a consequence, control of excitation efficiencies can be rationalized using the concept of interference between different excitation pathways associated with specific frequency combinations [1-3]. Such a frequency description inherently fails in the strong field regime that is required to obtain a significant non-equilibrium population in excited levels. Strong-field excitation is accompanied by the presence of Rabi cycles and dynamic Stark shift (DSS) of states, which implies that resonance conditions are not conserved during the excitation [4].

DSS effectively adds a degree of control freedom. Although control in a strong field is powerful, optimization of pulse shape becomes distinctly less intuitive. Nevertheless, early attempts achieved full inversion in an intuitive way in a three level systems making use of chirped laser pulses in a process involving a rapid adiabatic passage (RAP) mechanism [5, 6]. A systematic search combining RAP and a phase mask is found in Bruner et al. [7]. Modern optimization schemes often make use of learning algorithms with experimental feedback $[8,9]$, however, the interpretation of the results often remains difficult $[10,11]$. A general approach for obtaining population inversion implies adjustment of pulse parameters to compensate for DSS. This was shown both analytically $[12,13]$ and experimentally [13], also in combination with the use of a genetic algorithm [14].

One way to interpret the excitation in strong field uses diagonalization of the Schrödinger equation. In the resulting dressed state picture, levels split to the extent of the Rabi frequency during the interaction. With more than one Rabi cycle during an intense ultrashort pulse, this splitting becomes proportionally larger than the pulse bandwidth. The reality of such splitting was experimentally verified by $\mathrm{Wu}$ et. al. already in 1975 [15]. The spontaneous fluorescence 
spectrum from a strongly driven sodium vapor by a narrow band continuous wave laser revealed two side bands associated with the excitation strength $[15,16]$.

In recent years, Baumert and co-workers have found another implication of the dressed state picture in a strong field control [17-19]. They proposed the concept of selective population of dressed states (SPODS) and demonstrated the possibility to address individual dressed states in the excited system. For example, potassium (K) was excited to the intermediate state by a shaped pulse, while the same pulse caused a further two-photon ionization perturbatively. Using photoelectron spectroscopy and changing the temporal phase in the excitation pulse, they showed not only the presence of an Autler-Townes doublet, but also selective ionization of the upper (giving relative high energy electrons) or the lower of the two dressed states [1717, 19].

In this letter, we aimed at studying the control possibilities to achieve full inversion using offresonant excitation under conditions, where a perturbative picture does not permit any excitation. We do demonstrate both strong enhancement of the two-photon excitation rate as well as complete population inversion in rubidium. The enhancement of population transfer under far offresonant excitation conditions can be phenomenologically explained using the dressed state picture. While the photon-energies within the excitation field are significantly lower than both 5s$5 p$ and $5 p-5 d$ transition energies, our results suggest that the intermediate level is coupled with the ground and final levels via the lower and higher lying dressed states respectively. Effectively, the splitting of the intermediate level into two dressed states compensates for the energy difference between the laser field and both transitions ( $5 s-5 p$ and $5 p-5 d)$. In general, our results support the SPODS concept in an aspect that the (intermediate) level can be addressed via different dressed states during the excitation. 
To control the excitation efficiency to the $5 \mathrm{~d}$ rubidium level we block a certain frequency range in the spectrum. A consequence of blocking frequencies is the creation of pre-and post-transients in the pulse, which, as we show, have a pivotal role in the excitation process. In the case of intense pulses, these transient can generate a significant excited state population [20]. This was employed, for example, by Gürtler and van der Zande [21] in a special case of Rb atoms excited in Rydberg states and $\mathrm{THz}$ radiation, where the combination of Rabi-cycles and post transients resulted in an enhanced excitation efficiency at specific photon energies, even though, formally, the power spectrum of the resulting pulse lacks the required specific photon energies. In a more practical application, one now implements a scheme using absorption to create a coherent transient to seed LCLS, the X-ray Free Electron Laser in Stanford, using Bragg reflection on a diamond crystal to block a narrow frequency range in the keV photon energy range. [22]

We here present systematic studies in the population in the $5 \mathrm{~d}$ state in Rubidium as function of amplitude shaped intense pulses, and present a simple way to get full inversion in a three-level system or zero excitation of the final level with minimum adaptations of the pulse.

\section{Experiment}

In our experiment, rubidium was used as a model system. The population in the final $5 \mathrm{~d}$ state was probed by observing the second photon in the two-photon fluorescent cascade. The level diagram (see figure 1 (a)) involves the two intermediate $5 \mathrm{p}$ spin orbit states, $5 \mathrm{p}_{3 / 2}$ and $5 \mathrm{p}_{1 / 2}$, with excitation wavelengths $\lambda_{5 \mathrm{p} 3 / 2}=780.2 \mathrm{~nm}$ and $\lambda_{5 \mathrm{p} 1 / 2}=795 \mathrm{~nm}$, respectively. Although the strong field regime requires a description involving both spin-orbit states, the pathway to the $5 \mathrm{~d}$ states via the $5 \mathrm{p}_{3 / 2}$ dominates. For the excitation, amplified $40 \mathrm{fs}$ laser pulses were used with a repetition rate of $250 \mathrm{kHz}$, generated from a Coherent Reg A 9050 amplifier and centered at $796 \mathrm{~nm}$. The beam was focused in a cell, containing rubidium vapor, to a spot size of about $10 \mu \mathrm{m}$. The pulse energy after the shaper was approximately $0.4 \mu \mathrm{J}$. We used amplitude pulse shaping only, by blocking part of the spectrum in the Fourier plane. The fluorescence intensity was measured as a 
function of the spectrum block position. It is important to notice that the area, from which the fluorescence was collected, covered a range of laser intensities due to the short focal length and the Gaussian profile of the laser beam.

\section{Results and discussion}

The fluorescence intensity as a function of the spectral block position is shown in figure 2. In part (a) the excitation spectrum and the direction of the block scan are shown. The most remarkable observation is the large population enhancement for the spectrum block position close to the $\lambda_{5 \mathrm{p} 3 / 2}$ resonance (figure 2 (b)) at $780.2 \mathrm{~nm}$. This enhancement proves strong-field excitation, since in the perturbative limit, no population transfer is possible if all frequencies below or above the central energy $(778 \mathrm{~nm})$ are blocked [23]. The population transfer hardly increases when a spectral block is positioned near the second resonance at $\lambda_{\mathrm{fi}}=776 \mathrm{~nm}$. In fact, the minor peak, observed at $\lambda_{\mathrm{fi}}=776 \mathrm{~nm}$ is caused by the contribution of intermediate intensities. The enhancement at the resonances in perturbative limit using spectral block as control parameter has been reported and explained by Dudovich et al. [23].

To confirm that the enhancement of the excitation at $\lambda_{5 \mathrm{p} 3 / 2}$ resonance is the result of strong field effects, we have also performed experiments as a function of laser fluence (not shown). The reduction of the laser intensity causes the enhancement at the $\lambda_{5 \mathrm{p} 3 / 2}$ resonance $(780.2 \mathrm{~nm})$ to gradually disappear, representing a transition from the strong-field to the weak-field regime.

The experimental data are in satisfactory agreement with calculations (see figure 2). We solve numerically the time-dependent Schrödinger equation for rubidium while using an FFT method to transform the shaped pulse spectrum back to the time domain. For details see Ref [20]. We included always the two intermediate spin-orbit states $\left(5 p_{3 / 2}\right.$ and $\left.5 p_{1 / 2}\right)$, even when the $5 p_{3 / 2}$ state 
dominated the dynamics. We have used the laser intensity as optimization parameter. The experimental data were calibrated to fit the calculated populations at the level, corresponding to the excitation by transform-limited pulse. Optimal agreement between experiment and calculations was found in a range of laser intensities, associated with a Rabi frequency between 24 and $34 \mathrm{THz}$ (for the 5s-5p transition excited by an unshaped pulse). This intensity range lies within a first Rabi maximum in the final $5 \mathrm{~d}$ state population. The calculated absolute population in the final level varied from $40 \%$ to $80 \%$ respectively.

We will now discuss the dynamics of the system during the excitation. The calculated populations of the ground, intermediate and final levels are shown in figure 3 for conditions as in figure 2 for two positions of the spectrum block: at $780 \mathrm{~nm}$, where the maximum enhancement is observed (a), and at $781 \mathrm{~nm}$, where the population in the final state is reduced (b). Excitation by a pulse pre-transient creates almost complete coherence of the ground and intermediate $5 \mathrm{p}_{3 / 2}$ state at -90 fs before the main pulse arrives (figure 3). For both positions of the spectral block, approximately $50 \%$ of the population is transferred to the intermediate $5 \mathrm{p}_{3 / 2}$ state during excitation by a pulse pre-transient. During the intense pulse rapid changes of the populations are observed, associated with Rabi oscillations. The coupling of the ground and intermediate states by the pretransient defines relative phase between these states. This phase difference and the populations form the initial conditions for the excitation during the strong pulse. Depending on the exact position of the spectral block relatively to the resonance a different phase results from the pre-transient. In our case the calculated relative phase (not shown) between the $5 p_{3 / 2}$ and the ground state changes with $\pi$ for the spectrum block positioned at different sides from the resonance frequency (at $780 \mathrm{~nm}$ and at $781 \mathrm{~nm}$ ). The $\pi$ difference explains the opposite direction of the population transfer between the ground and the $5 \mathrm{p}_{3 / 2}$ state triggered by Rabi oscillations (after -90 fs) (see figure 3). 
A Wigner distribution of the pulse shows that the pretransient has a narrow spectral distribution around the frequency, where the spectrum block was applied. The intense part of the pulse has a near Gaussian spectral profile around the central frequency of the unshaped pulse (provided the block is in the wing of the spectral distribution). Applying the spectral block at the position red or blue detuned from the resonance results in the excitation by a pretransient whith the carrier frequency red or blue detuned from the resonant transition; consequently, the phase difference of $\pi$ in the $5 \mathrm{p}_{3 / 2}$ state arises.

The population transfer during off-resonant excitation can be rationalized using a dressed state picture as illustrated in figure 1(b). An efficient population transfer to the final level combines resonant excitation conditions using the transients followed by a strong pulse interaction, in which the momentary frequency in the laser pulse follows the energy of the transitions. The low energy of the laser carrier frequency suggests that the intermediate level is coupled to the ground and final level via the $5 \mathrm{p}_{3 / 2}$ lower and higher lying dressed states, respectively (see figure 1 (b)). Effectively, the splitting of the intermediate level into two dressed states compensates for the insufficient photon energies to couple the states involved. Our results suggest that in the excitation individual dressed states play a role, which agrees with the SPODS concept that proposes the possibility to address individual dressed states in the system $[17,18]$. However, the interpretation of the excitation in terms of population of individual dressed states may not always be valid. Such a description cannot explain the population transfer from the intermediate to the final level in our experiment. Of course, explanations in terms of population of individual dressed states are ambiguous, as the dressed state energies reflect the rate of change in the level populations.

In the following, we demonstrate analytically a simple way to achieve complete population inversion in rubidium. The calculated results are shown in figure 4 . The differences with the previous conditions (as in figure 2 (a)) are the shift of the central frequency of the excitation 
spectrum to $778 \mathrm{~nm}$. As shown in figure 4 (b), when the spectrum block is located at $778 \mathrm{~nm}$, $97.5 \%$ of the population is transferred to the final state. We note that a large population transfer above $95 \%$ could be achieved in a wide range of parameters: laser central frequency and Rabi frequency could be varied within several $\mathrm{nm}$ around $778 \mathrm{~nm}$ and between 22 to $26 \mathrm{THz}$ respectively. Due to the limitations in the experimental settings (the laser system did not allow to shift the position of the laser central wavelength to shorter wave lengths), we were not able to confirm this theoretical finding experimentally.

In figure 5 the time evolution of the state populations is shown for two positions of spectral block: at $778 \mathrm{~nm}$ (maximum enhancement) and at $780.4 \mathrm{~nm}$ (zero population transfer). Due to the strong reduction in the pulse intensity (more then half of the spectrum is blocked), the period of the Rabi oscillations is larger compared to figure 3. Similar to the results in figure 3, the spectral block position controls the phase of the Rabi oscillations: the rapid changes in the population in the $5 \mathrm{p}_{3 / 2}$ state have opposite direction for different position of the spectrum block. As in the situation of Fig. 3, our calculations give a $\pi$-phase difference in the $5 \mathrm{p}_{3 / 2}$ level for the two positions.

Due to the lower strength of the excitation field the energy shifts are much smaller then in the previous experiment; hence, it becomes more ambiguous to rationalize the efficient population transfer using a dressed state picture. However, the results of this experiment are consistent with the previously discussed mechanism of the population transfer. We suggested above that the dressed states effectively create momentary resonant conditions during the intense laser pulse. In this experiment, the laser central frequency during the intense pulse is slightly lower in energy then the unperturbed resonant transitions, hence, small level shifts are sufficient to fulfill momentary resonant conditions. 


\section{Conclusions}

In conclusion, we have demonstrated an enhancement of the population transfer in Rubidium during an off-resonant two photon excitation in a strong field. The enhancement is achieved by using the pre-transient in the excitation pulse, created by amplitude shaping. The underlying physical mechanism in the proposed control scheme can be described as manipulation of the initial phase of Rabi oscillations by creating a specific phase and population distribution in the system before the Rabi oscillations start. In the experiment $70 \%$ of the population could be created in the final state, while with additional variation of parameters, calculations predict complete population inversion.

\section{Acknowledgements}

We acknowledge the technical support of T. Toonen, A. van Etteger and the financial support of FOM, NWO and EC FP7 ERC Grant agreement No 257280 (Femtomagnetism). We greatly acknowledge fruitful discussions with T. Baumert. 
1. Chen Z, Brumer P and Shapiro M 1992 Chem. Phys. Lett. 198, 498-504.

2. Meshulach D and Silberberg Y 1999 Phys. Rev. A 60 1287-92.

3. Clow S and Weinacht T 2010 Phys. Rev. A 82023411.

4. Trallero-Herrero C and Weinacht T C 2007 Phys. Rev. A 75063401.

5. Broers B, van den Heuvell H B V L and Noordam L D 1992 Phys. Rev. Lett. 69 2062-5.

6. Maas D J, Rella C W, Antoine P, Toma E S and Noordam L D 1999 Phys. Rev. A 59 1374-81.

7. Bruner B D, Suchowski H, Vitanov N V and Silberberg Y 2010 Phys. Rev. A 81 063410.

8. Judson R S and Rabitz H 1992 Phys. Rev. Lett. 68 1500-3.

9. Assion A, Baumert T, Bergt M, Brixner T, Kiefer B, Seyfried V, Strehle M and Gerber G 1998 Science 282 919-22.

10. Bartels R A, Murnane M M, Kapteyn H C, Christov I and Rabitz H 2004 Phys. Rev. A 70043404.

11. Cardoza D, Baertschy M and Weinaht T C 2005 J. Chem. Phys. 123074315.

12. Trallero-Herrero C, Cardoza D, Weinaht T C and Cohen J L 2005 Phys. Rev. A 71013423.

13. Lee S, Lim J, Ahn J, Hakobyan V and Guerin S 2010 Phys. Rev. A 82023408 .

14. Trallero-Herrero C, Cohen J L and Weinaht T C 2006 Phys. Rev. Lett. 96063603.

15. Wu F Y, Grove R E and Ezekiel S 1975 Phys. Rev. Lett. 35 1426-9.

16. Grove R E, Wu F Y and Ezekiel S 1977 Phys. Rev. A 15 227-33.

17. Wollenhaupt M, Liese D, Präkelt A, Sarpe-Tudoran C and Baumert T 2006 Chem Phys. Lett. 419 184-190.

18. Wollenhaupt M, Bayer T, Krug M, Sarpe-Tudoran C and Baumert T $2007 \mathrm{~J}$. Phys.: Conf. Series $\mathbf{8 8} 012053$.

19. Wollenhaupt M, Assion A, Bazhan O, Horn Ch, Liese D, Sarpe-Tudoran Ch, Winter M, Baumert T 2003 Phys. Rev. A 68015401.

20. Meijer A S, Kimel A V, Rasing Th and van der Zande W J 2008 Phys. Rev. A 78 053403.

21. Gürtler A and van der Zande W J 2004 Phys. Rev. Lett. 93153002.

22. Amann J, Berg W, Blank V, Decker F J, Ding Y, Emma P, Feng Y, Frisch J at al 2012 Nature Photonics.

23. Dudovich N, Dayan B, Gallagher Faeder S M and Silberberg Y 2001 Phys. Rev. Lett. 86 47-50. 
(a)

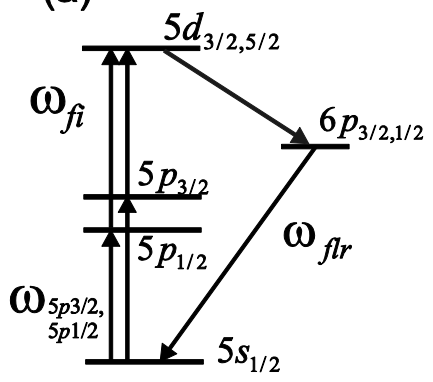

(b)

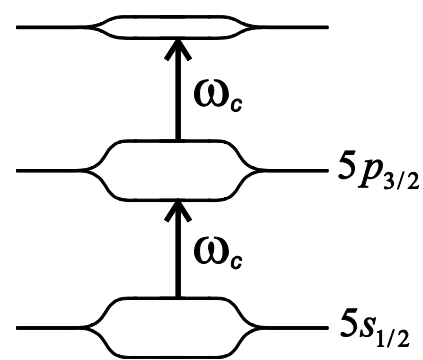

Figure1. (a) The energy level scheme of Rubidium. The $5 \mathrm{~d}$ level is excited via a two-photon process. The resonant transitions are at $\lambda_{5 \mathrm{~s}-5 \mathrm{p} 3 / 2}=780.2 \mathrm{~nm}$ and $\lambda_{5 \mathrm{~s}-5 \mathrm{p} 1 / 2}=776.0 \mathrm{~nm}$. The atoms decay spontaneously from the $5 \mathrm{~d}$ to the $5 \mathrm{~s}$ state via the $6 \mathrm{p}$ state. The population in the $5 \mathrm{~d}$ state is determined by observing fluorescence at around $420 \mathrm{~nm}$. (b) Schematic illustration of the excitation to the final $5 \mathrm{~d}$ level using the dressed state picture. The levels split during intense pulse making an excitation of excited states by low energy photons possible. 

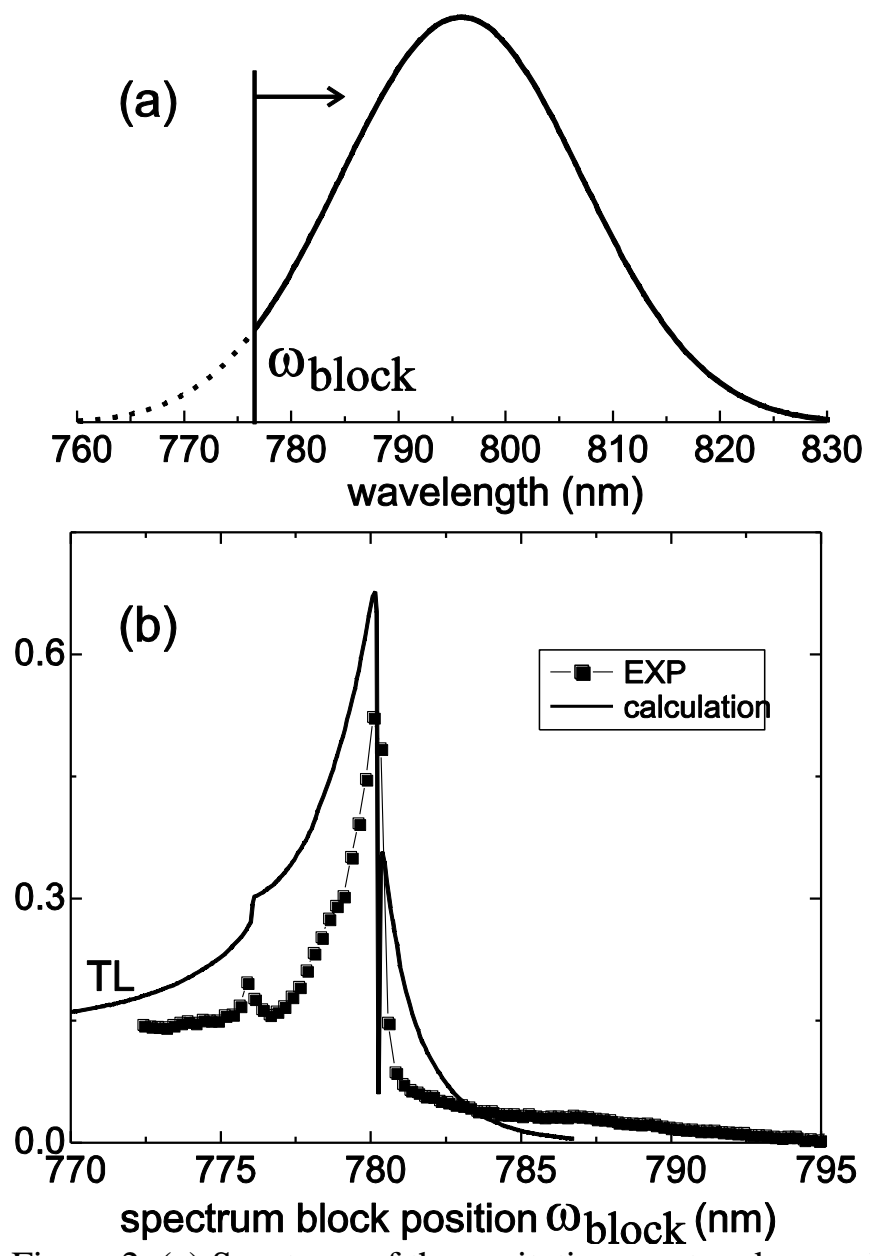

Figure 2. (a) Spectrum of the excitation, centered around $795 \mathrm{~nm}$. Part of the spectrum is blocked and the position of the block is scanned. (b) - Fluorescence intensity as a function of the spectrum block position. TL indicates the signal during the excitation by an unshaped pulse. 


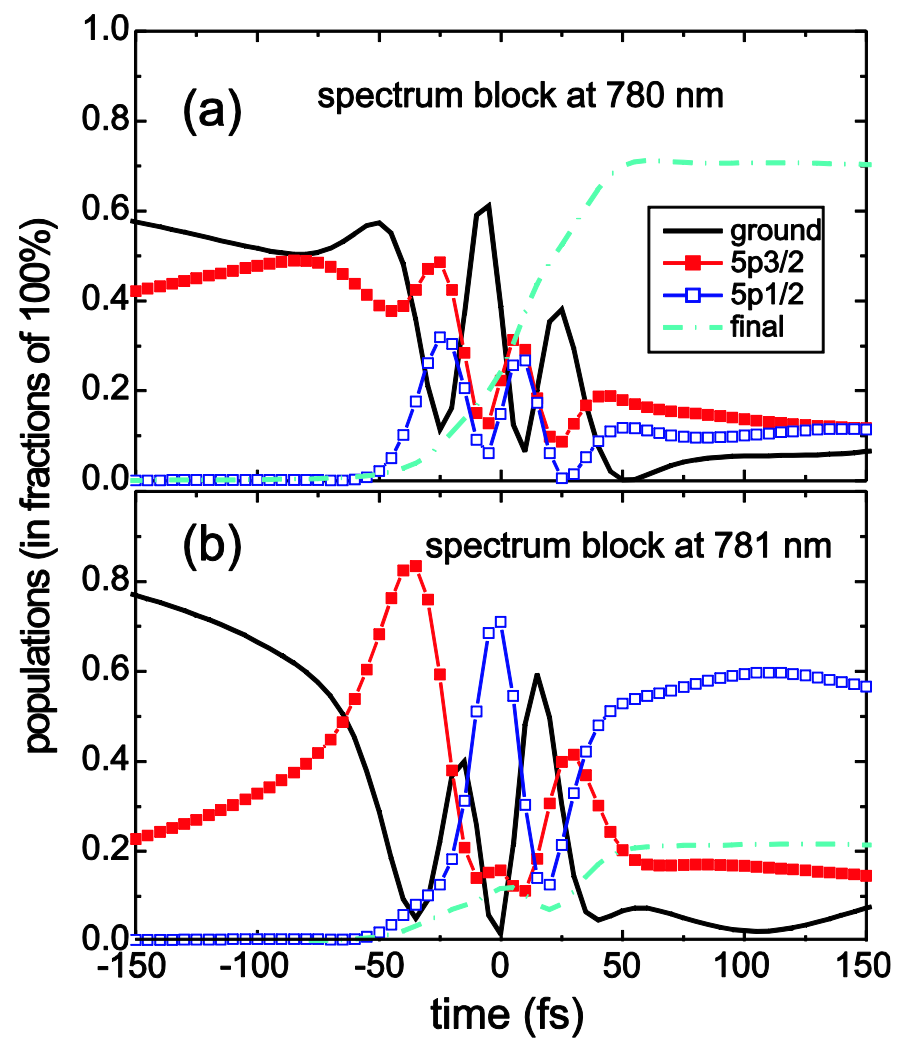

Figure 3. (Color online) Calculated time-dependencies of populations of the ground, intermediate and final states for the conditions as in Fig. 2. (a) Spectrum block is positioned at $780 \mathrm{~nm}$ where the maximum of enhancement is observed. (b) Spectrum block is positioned at $781 \mathrm{~nm}$. 


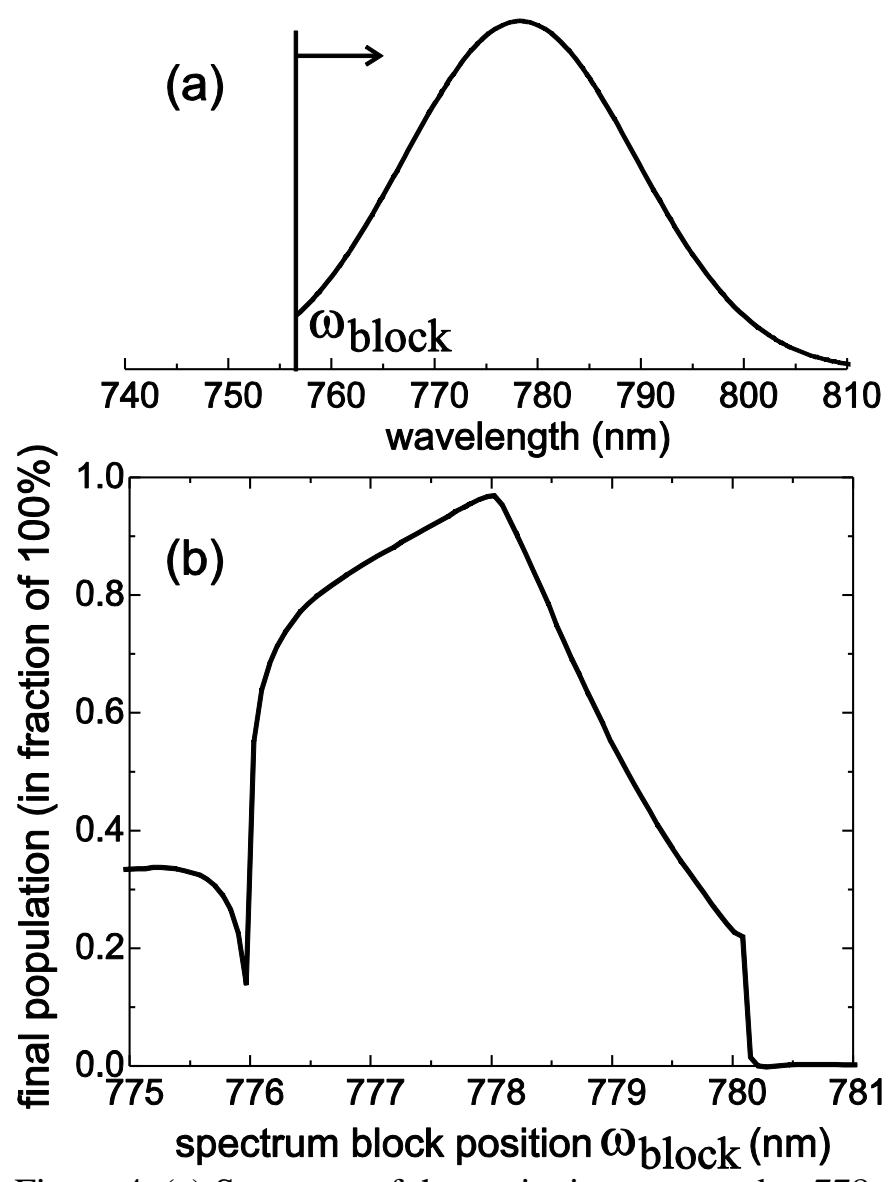

Figure 4. (a) Spectrum of the excitation, centered at $778 \mathrm{~nm}$. Part of the spectrum is blocked and the position of the block is scanned. (b) Calculated population in the final $5 \mathrm{~d}$ level as a function of spectrum block position. At $778 \mathrm{~nm}$ the population reaches $97.4 \%$. 


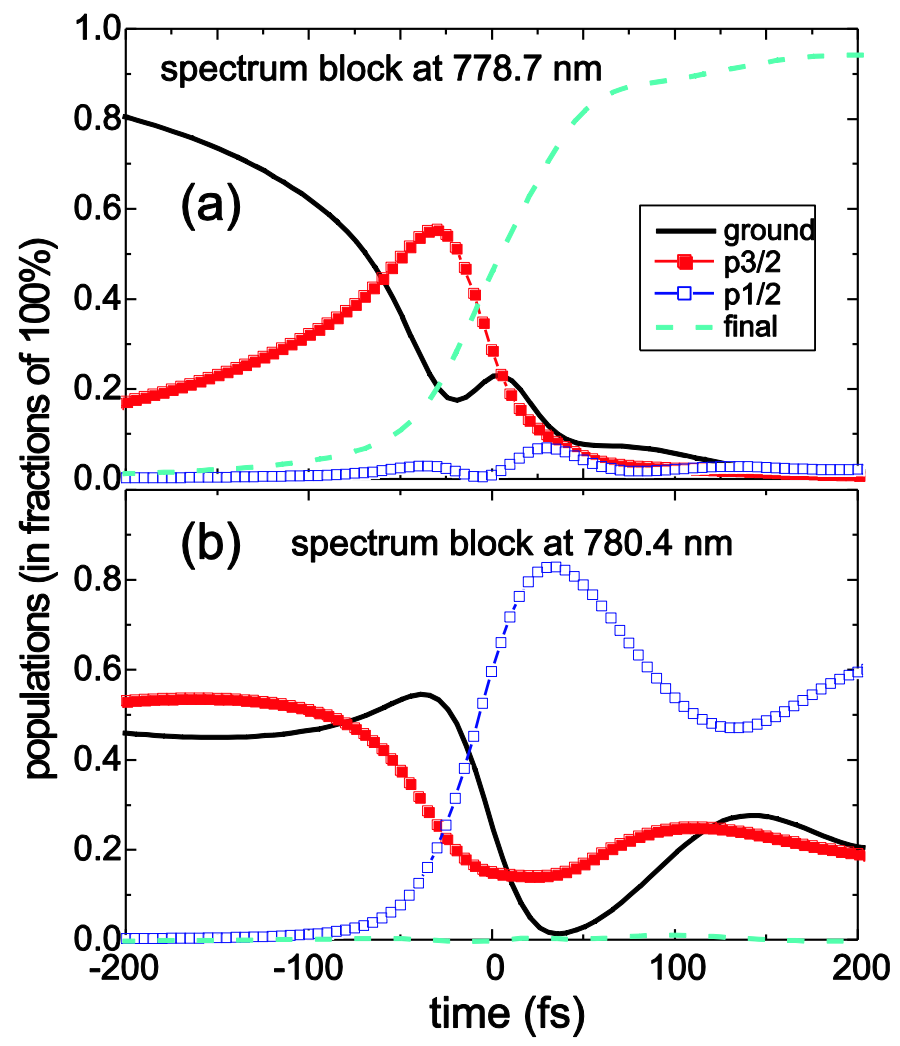

Figure 5. (Color online) Calculated time-dependencies of populations of the ground, intermediate and final states for the conditions as in Fig 4. (a) Spectrum block is positioned at $778 \mathrm{~nm}$ where the maximum of enhancement is observed. (b) Spectrum block is positioned at $780.4 \mathrm{~nm}$ 